\title{
A Survey Paper on Wearable Sensors based
}

\section{Fall Detection}

\author{
Trupti Prajapati \\ Student, M.Tech \\ Computer Engineering \\ CSPIT, Changa
}

\author{
Nikita Bhatt \\ Assistant Professor \\ $U$ and P.U. Patel Dept. of \\ Computer Engineering \\ CSPIT, Changa
}

\author{
Darshana Mistry \\ Technical Associate \\ Software Training \\ eiTRA, Ahmedabad
}

\begin{abstract}
Falling is often accepted as a natural part of the aging process Elderly people are typically, more unsteady and frailer. Thus are more likely to fall and be injured than younger individuals. Falls can have a serious affect on both the quality of life of elder people and on health as well as social care costs. In this paper, we give a comprehensive survey of different wearable sensors for fall detection and their underlying algorithms and comparing their strengths and weaknesses. Conclusion is derived with some discussions on techniques of fall detection and pros and cons to wear wearable device for fall detection.
\end{abstract}

\section{Keywords}

Wearable sensor, machine learning, fall detection, elder people, ADL

\section{INTRODUCTION}

We Currently $7 \%$ to $8 \%$ Elderly peoples live alone because of some social reason and property crime and violent crime rate increases with elderly people who live alone because they do not have enough strength to take care about him or her. [1]

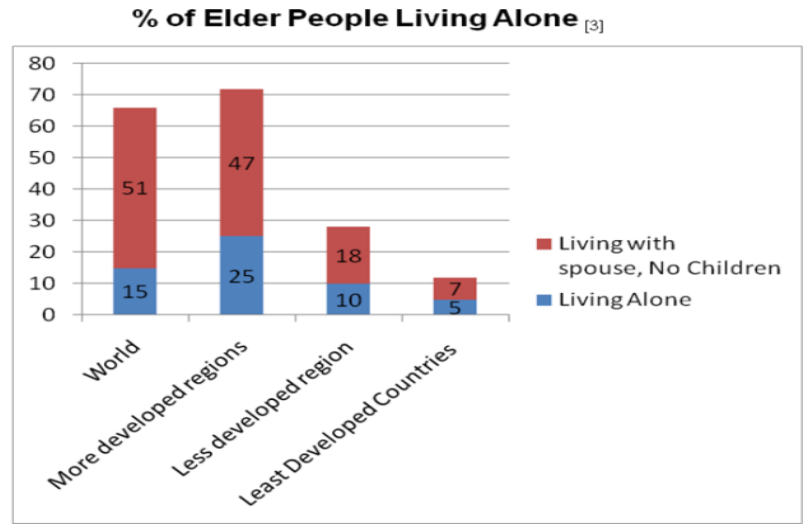

Fig 1: Statistic of elder people living alone

Therefore, a fall accident detection system, which can automatically detect the fall accident and call for help, is very important for elderly people, especially for those living alone. With wearable sensors attached to the elderly people, falls can be automatically detected. Alert messages can be generated and delivered to the predetermined contacts.

Fall can be detected using wearable sensor, vision based and ambient sensor. All three technique have own advantages and disadvantages based on accuracy and time. But in this survey, mainly focus on wearable based fall detection. For many years, fall detection is interesting subject for research and commercial view.

The remaining section of this paper is organized as follows: Section 2 contains wearable sensors use for fall detection. In section 3, fall detection using machine learning algorithm section 4 contains comparison between fall detection techniques, section 5 contains reviews previous research finding and section 6 contains conclusion.

\section{WEARABLE SENSORS USED IN FALL DETECTION}

Many sensors use for fall detection like accelerometer sensor, heart rate sensor, Gyroscope sensor, magnetometer sensor and microphone.

\subsection{Accelerometer}

Acceleration is the measurement of the change in velocity, or speed divided by time. An accelerator looks like a simple circuit for some larger electronic device. Acceleration is measured in $(\mathrm{m} / \mathrm{s}) / \mathrm{s}$. This angle is very useful to detect fall.

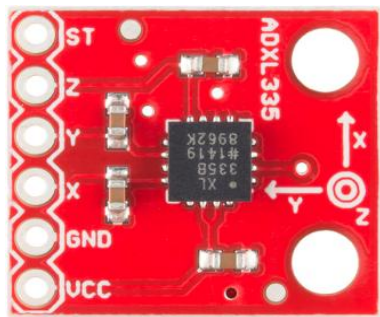

Fig 2: Hardware of Triple Axis Accelerometer [2]

$\mathrm{X}, \mathrm{Y}, \mathrm{Z}$ are the three axis of the accelerometer to detect to detect the motion and location of the body of the subject. A fall is detected when the negative acceleration is suddenly increased due to the change in orientation from upright to lying position.

\subsection{Gyroscope Sensor}

Gyro sensors give angular velocity. Angular velocity is the change in rotational angle per unit of time. Unit of angular velocity is deg/s (degrees per second).

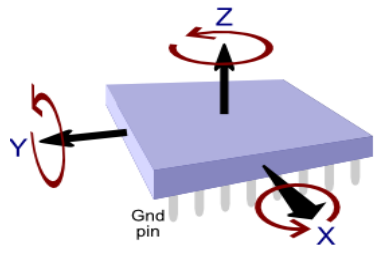

Fig 3: Axis Gyroscope Sensor 
[G.M. Lyons]Author has proposed an algorithm that only depends on one biaxial gyroscope. Using simulated-falls performed by young volunteers under supervised conditions onto crash mats and ADL performed by elderly subjects; This is done by setting thresholds for the gyroscope. From the gyroscope pitch and roll angular velocity are obtained, if these values pass the thresholds then an alarm is triggered to warn of a fall. This system has shown high accuracy for fall detection.

Three thresholds were identified so that a fall could be distinguished from an ADL:

Fall detection threshold

1. Resultant angular velocity $>3.1 \mathrm{rads} / \mathrm{s}$

2. Resultant angular acceleration $>0.05 \mathrm{rads} / \mathrm{s}$

3. Trank-angle $>0.59$

Results show that falls can be distinguished from ADL with $100 \%$ accuracy, for a total data set of 480 movements. A falldetection strategy has been proposed and verified with $100 \%$ specificity obtained over a total of 240 ADL events recorded [3].

\subsection{Heart Rate System}

A heart rate monitor is a subjective monitoring device that allows one to measure one's heart rate in real time or record the heart rate for later study. Some authors use fusion sensor data including heart rate data to detect fall. Accelerometer data and heart rate is used to detect both abnormal cardiac accelerations and abnormal movement of human. Heart rate data also used to reduce false alarm.

\subsection{Magnetometer Sensor}

A magnetometer is used to measure a magnetic field associated with the user's change of orientation.

\subsection{Microphone}

A microphone is a use to receive and analyze sound like human screaming and crying sounds. Fusions of accelerometer sensor and microphone data are help to reduce false alarm and also help to chance to increases true alarm for fall detection.

[Ram David] invented fall detection system using a combination of accelerometer, audio input and magnetometer sensor [4].

\section{FALL DETECTION USING MACHINE LEARNING ALGORITHM}

Machine learning is a type of artificial intelligence (AI) that investigates the ability of machines to understand data.

\subsection{Multilayer Perceptron (MLP)}

Please MLP is a feed forward artificial neural network. A MLP have input layer (set of source node), hidden layer (computational node) and output layer (output node).Artificial neural network is most effective learning technique for complex problems like learning to translate real-world data. MLP is mostly used to predict class of static, dynamic and fall activity.

[Hamideh] Proposed structure of MLP with 28 input layer and 6 hidden layer based on trial and error experiment to classify falling pattern from ADL. Data of input layer has been come from feature extraction (feature extraction done using data of Accelerometer sensor. MLP structure used Tansing transfer function for classification. Result of fall detection showed that falls can be distinguished from ADLwith a specificity of $91.07 \%$, accuracy of $91.6 \%$, sensitivity of $92.03 \%$, and from a total of 250 test data.

Future work - accuracy can be improved using tuning of its parameters [5].
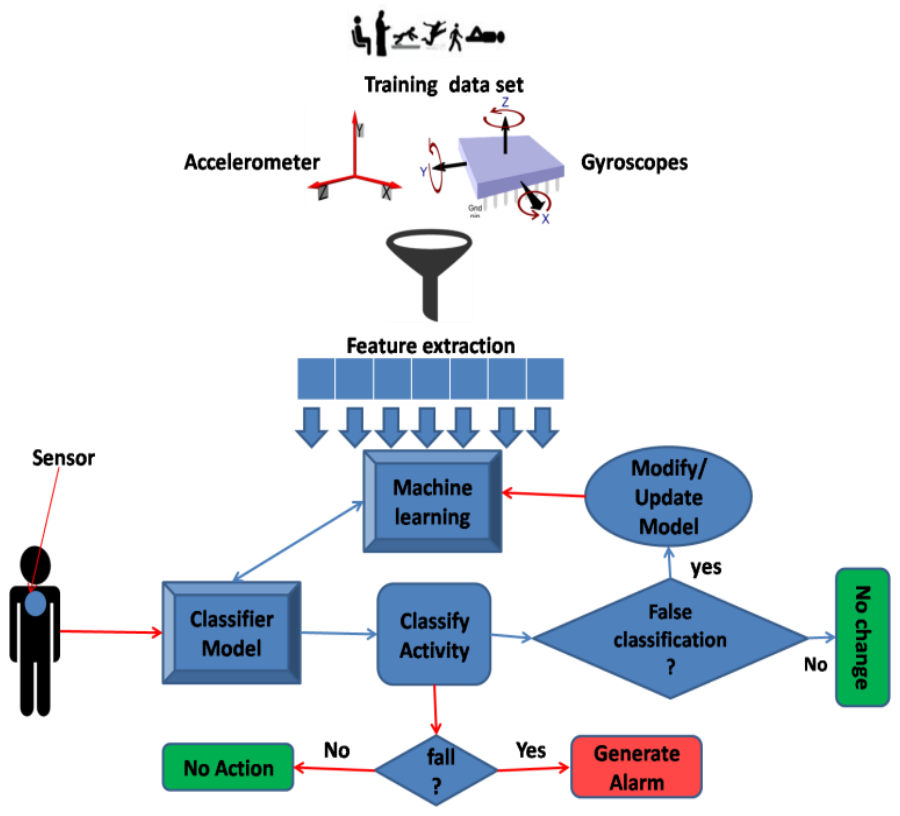

Fig 4: Classification Structure of Fall Detection using Accelerometer Sensor and Gyroscopes Sensor

\subsection{Navie Bayes}

[Xiuxin Yang] implemented fall detection using wearable sun-SPOT sensor. Sun-SPOT sensor has been made using accelerometer. Two sun-SPOT sensor attached to chest and thigh for fall detection using Navie Bayes algorithm. Navie Bayes is compared with other machine learning algorithm such that Support Vector Machine, C4.5 (J48), neural network. Based on result, Navie Bayes have good accuracy then another 3 machine learning algorithm. $88.06 \%$ instances were correctly classified. 58 instances was incorrectly classify out of 486 . Using Navie Bayes, sensitivity is $87.5 \%$ with less false negative detection for fall detection [6].

\subsection{Support Vector Machine (SVM)}

Support Vector Machine (SVM) is a kind of supervised learning method that simultaneously minimizes the empirical classification error and maximizes the geometric margin. In this classification method there is a set of training example that belong to one of two categories, then SVM predicts that new example fall in to which category.

[Omar Aziz] studied that machine-learning pre-impact fall detection method - support vector machines (SVM) are better than traditional threshold-based methods for better adaptability and reliability. The system used a wide variety of fall and daily activity scenarios to more rigorously test the accuracy of our SVM algorithm across a combination of varying lead times and window sizes, using a single waist mounted tri-axial accelerometer and gyroscope. The system provided at least $95 \%$ sensitivity and at least $90 \%$ specificity for combinations of window size from $0.125-1 \mathrm{~s}$ and lead time from $0.0625-0.1875 \mathrm{~s}$. By performing trial and error method, lead times $0.25 \mathrm{~s}$ or greater, sensitivity and specificity varied dramatically with choice of window size, indicating poor 
robustness of the classification performance. Therefore, the use of a target lead time around $0.1875 \mathrm{~s}$ or less, and window size $1 \mathrm{~s}$ or less for robust pre-impact fall detection [7].

\section{REVIEW PREVIOUS RESEARCH FINDINGS}

[Jung Keun Lee] devised two techniques to detect fall. There were two classification cases (fall vs. non-fall and fall vs. ADL). Near-fall and ADL are included in Non-fall. First, vertical velocity threshold based fall detection using acceleration sensor and gyroscope sensor. Fall was detected, if vertical velocity exceeds a threshold. Second, acceleration magnitude threshold based fall detection using acceleration sensor. Vertical velocity and acceleration magnitude had approximately same accuracy for Fall vs. ADL (sensitivity $97.4 \%$ and specificity $99.4 \%$ ). Vertical velocity based fall detection has high accuracy than Acceleration magnitude detection for Fall vs. Non-fall classification. [8]

[Ahmet] developed fall detection with wearable sensors attached to subject's body at six different positions. Mainly, they used accelerometer, gyroscope, and magnetometer. They compared different machine learning algorithm for fall detection: the k-nearest neighbour (k-NN) classifier, least squares method (LSM), support vector machines (SVM), Bayesian decision making (BDM), dynamic time warping (DTW), and artificial neural networks (ANNs). Before data pre-processing he used sliding window technique to partition data. They decided $5 \mathrm{sec}$ time duration of window. They concluded, the k-nearest neighbour (k-NN) classifier and least squares method (LSM) had best result at above 99\% sensitivity, accuracy and specificity.[9]

[Shaoming]Author provided the use of Support Vector Machines (SVM) and a tri-axial accelerometer to detect falls. This is done by generating feature vectors derived from time domain characteristics and then applying feature selection. The SVM focuses on feature vectors to determine if the activity is a fall or an everyday event. Feature selection and pattern classification are used to maximize the recognition accuracy.Result: The overall system was tested and results showed that all falls could be detected with an average leadtime of $203 \mathrm{~ms}$ before impact, and no false alarm occurred. The proposed system will lead to potential applications for preventing or reducing fall-related injuries [10]

[John Lach]An author has devised an algorithm that depends on the use of both accelerometers and gyroscopes. They defined falling as an unintentional change in posture to the lying posture. Thresholds are set based on testing done by measuring the acceleration and angular velocity. Accordingly, if those thresholds are reached, then the system assumes that the change in posture was unintentional and a fall has occurred [11]. Author's algorithm, coupled with accelerometers and gyroscopes, reduces both false positives (sitting down fast) and false negatives (falling on stairs), while improving fall detection accuracy. In addition, solution features low computational cost and real- time response.

Limitation: Method of author faced difficulties in separating jumping into bed and falling against wall with a seated posture. To distinguish these activities, context information (environmental/physiological) can be exploited

Future work: For detecting jumping into bed used the localization method [11].
Table 1: Comparison of Different Algorithm used in fall Detection

\begin{tabular}{|c|c|c|}
\hline $\begin{array}{l}\text { Author } \\
\text { Algorithm } \\
\text { Name }\end{array}$ & Sensors & Result \\
\hline $\begin{array}{l}\text { [12] } \\
\text { Chih-Ning } \\
\text { Huang }\end{array}$ & $\begin{array}{c}\text { Tri-axial } \\
\text { accelerometer }\end{array}$ & $\begin{array}{l}2.46 \% \text { probability of falls } \\
97.54 \% \text { probability of ADLs }\end{array}$ \\
\hline \multirow[t]{2}{*}{$\begin{array}{l}\text { Throsold } \\
\text { based fall } \\
\text { detection }\end{array}$} & \multirow[t]{2}{*}{$\begin{array}{l}\text { Position: left } \\
\text { side of waist }\end{array}$} & $\begin{array}{l}\text { For all ADLs, } \\
8.55 \% \text { probability of false } \\
\text { alarms, } 17.95 \% \text { probability } \\
\text { of normal falls and } 73.5 \% \\
\text { probability of ADLs. }\end{array}$ \\
\hline & & $\begin{array}{l}\text { Limitation: The false alarms } \\
\text { happened on lying on the bed } \\
\text { with fast speed and going up } \\
\text { and down stairs with normal } \\
\text { speed which are recognized } \\
\text { as normal falls. }\end{array}$ \\
\hline \multirow{3}{*}{$\begin{array}{l}\text { [13] } \\
\text { Changhong } \\
\text { Wang }\end{array}$} & \multirow{5}{*}{$\begin{array}{l}\text { Tri-axial } \\
\text { accelerometer, } \\
\text { barometric and } \\
\text { pressure } \\
\text { signals }\end{array}$} & $95.9 \%$ Accuracy \\
\hline & & 96.7\% Sensitivity \\
\hline & & $96.9 \%$ Specificity \\
\hline \multirow{2}{*}{$\begin{array}{l}\text { Throsold } \\
\text { based fall } \\
\text { detection }\end{array}$} & & $\begin{array}{l}10.9 \% \text { Reduction power } \\
\text { consumption }\end{array}$ \\
\hline & & $\begin{array}{l}\text { Pinpoint: focused on low- } \\
\text { power algorithms for } \\
\text { wearable devices with an } \\
\text { accelerometer }\end{array}$ \\
\hline $\begin{array}{l}{[14]} \\
\text { Lina Tong } \\
\text { hidden } \\
\text { Markov } \\
\text { model } \\
\text { (HMM) }\end{array}$ & $\begin{array}{c}\text { Tri-Axial } \\
\text { accelerometer }\end{array}$ & $\begin{array}{l}100 \% \text { Accuracy predict falls } \\
\text { in } 200 \sim 400 \text { ms before the } \\
\text { impact and can also } \\
\text { distinguish fall events. } \\
\text { Target: Simulation on } \\
\text { human activity data set on } \\
\text { young people }\end{array}$ \\
\hline \multirow{11}{*}{$\begin{array}{l}\text { Constructing } \\
\text { Cascade- } \\
\text { AdaBoost- }\end{array}$} & \multirow{11}{*}{$\begin{array}{l}\text { Tri-axial } \\
\text { accelerometer } \\
\text { Position: } \\
\text { chest and } \\
\text { waist }\end{array}$} & Chest \\
\hline & & AR-98.23\% \\
\hline & & DR-88\% \\
\hline & & FAR-1.27\% \\
\hline & & Waist \\
\hline & & AR-98.48\% \\
\hline & & DR-78.67\% \\
\hline & & FAR- $0.53 \%$ \\
\hline & & AR:- Accuracy rate \\
\hline & & DR:- Detection rate \\
\hline & & FAR:- False alarm rate \\
\hline
\end{tabular}




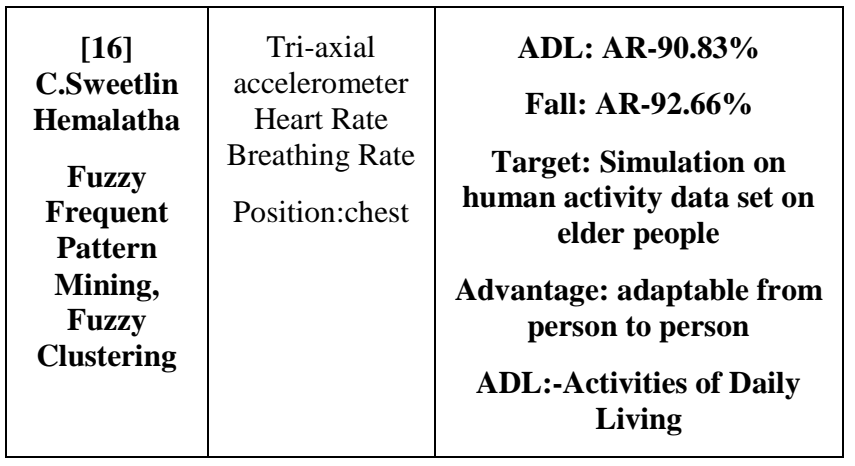

\section{CONCLUSIONS}

We have reviewed different techniques for the detection of a fall event using wearable sensors. Accelerometer sensor is mandatory to detect fall. But, detect fall using only accelerometer sensor gives more false alarms. To detect fall with accuracy, fusion of different sensor has been used. The biggest advantage remains the cost efficiency of wearable devices. Design setup and Installation is very comfort. So, the devices are comparatively easy to control. But, People have to continuously attach with sensor and wearable sensor is Intruder can access data from sensor. Such disadvantages make wearable devices an unfavorable choice for the elderly. In the future, it can be future extend with detailed survey of different types of fall (forward, backward, right and left) and survey of fall detection with vision based system using image processing and machine learning.

\section{REFERENCES}

[1] http://www.un.org/en/development/desa/population/publi cations/pdf/ageing/WorldPopulationAgeing2013.pdf

[2] https://www.sparkfun.com/products/9269

[3] A.K. Bourke, G.M. Lyons, "A threshold-based falldetection algorithm using a bi-axial gyroscope sensor", Medical Engineering \& Physics, Volume 30, Issue 1, Pages 84-90, January 2008.

[4] Leonid Baryudin, Ram David Adva Fish, Evgenia Goldshtein, Henry Messenger, Soroush Salehian Dardashti, "Fall Detection System Using a Combination of Accelerometer, Audio Input and Magnetometer",US 08843101 B2 Patent Summary.

[5] Hamideh Kerdegari, Khairulmizam Samsudin , Abdul Rahman Ramli \& Saeid Mokaram, "Development of wearable human fall detection system using multilayer perceptron neural network" International Journal of Computational Intelligence Systems, Pages 127-136, January 2013.

[6] Xiuxin Yang, Anh Dinh, Li Chen, “A wearable real-time fall detector based on Naive Bayes classifier" Electrical and Computer Engineering (CCECE), 2010 23rd Canadian Conference, Pages 1-4, May 2010.

[7] Omar Aziz, Colin M. Russell, Edward J. Park, Senior Member, IEEE, and Stephen N. Robinovitch, "The
Effect of Window Size and Lead Time on Pre-Impact Fall Detection Accuracy Using Support Vector Machine Analysis of Waist Mounted Inertial Sensor" Engineering in Medicine and Biology Society (EMBC), 2014 36th Annual International Conference of the IEEE, Pages 3033, August 2014

[8] Jung Keun Lee, Stephen N. Robinovitch, and Edward J. Park, "Inertial Sensing-Based Pre-Impact Detection of Falls Involving Near-Fall Scenarios" Neural Systems and Rehabilitation Engineering, IEEE Transactions on (Volume:23, Issue:2), Pages 258-266, September 2014.

[9] Özdemir, A.T.; Barshan, "Detecting Falls with Wearable Sensors Using Machine Learning Techniques" Sensors 2014, 14, 10691-10708.

[10] Shaoming Shan and Tao Yuan, "A wearable pre-impact fall detector using feature selection and Support Vector Machine" Signal Processing (ICSP), 2010 IEEE 10th International Conference, Pages 1686 - 1689, October 2010.

[11] Qiang Li, John A. Stankovic, Mark A. Hanson, Adam T. Barth, John Lach, "Accurate, fast fall detection using gyroscopes and accelerometer- derived posture information" Wearable and Implantable Body Sensor Networks, 2009. BSN 2009. Sixth International Workshop, Pages 138-143, June 2009.

[12] Huang, C.-N.; Chan, C.-T."A ZigBee-Based LocationAware Fall Detection System for Improving Elderly Telecare". Int. J. Environ. Res. Public Health 2014, 11, 4233-4248.

[13] Changhong Wang, Narayanan M.R. Lord S.R. Redmond S.J. Lovell N.H. "A low-power fall detection algorithm based on triaxial acceleration and barometric pressure" Engineering in Medicine and Biology Society (EMBC), 2014 36th Annual International Conference of the IEEE, Pages 570-573, August 2014.

[14] Lina Tong, Quanjun Song, Yunjian Ge, Ming Liu, "HMM-Based Human Fall Detection and Prediction Method Using Tri-Axial Accelerometer" Sensors Journal, IEEE (Volume: 13, Issue: 5), Pages 1849 1856, February 2013.

[15] Zhenyu Chen, Yiqiang Chen, Lisha Hu, Shuangquan Wang, Xinlong Jiang, "Leveraging Two-Stage Weighted ELM for Multimodal Wearables Based Fall Detection" Springer Proceedings of ELM-2014 Volume 2, Proceedings in Adaptation, Learning and Optimization Volume 4, Pages 161-168, 2015.

[16] J. Surana, C.S. Hemalatha, V. Vaidehi, S.A. Palavesam, M.J.A. Khan, "Adaptive learning based human activity and fall detection using fuzzy frequent pattern mining", Recent Trends in Information Technology (ICRTIT), 2013 International Conference, Pages 744-749, July 2013. 